Cette emprise du sol par l' " armature végétale " est le correctif physiologique de son érosion, elle est nécessairement progressive et suspend cette érosion.

L'adaptation de l' " armature " aux divers milieux géographiques, est un fait d'évolution physiologique, de " plasticité végétale ". Il est déterminé surtout par les conditions dans lesquelles le sol est approvisionné d'eau par les courants atmosphériques.

Suivant cette répartition, d'ordré éminemment géographique, l' " armature " groupe ses espèces en " Associatıons naturelles " : celles-ci évoluent par élimination des individus inaptes à lutter ou à assurer la prospérité des types en voie de proéminence. Ces types protègent à leur tour les espèces qui leur restent subordonnées. L'ensemble évolue et prospère par une sorte de "bénéfice mutuel "; 1l a comme terme, dans les régions hydro-climatıques, l'association à espèces ligneuses dominantes, la Forêt.

Dans la Forêt, les organes aériens de l'arbre fonctionnent comme écrans condensateurs des eaux atmosphériques : ils évaporent directement la plus grande partie de la pluie reçue. Les eaux condensées arrivent progressivement au sol; elles s'emmagasinent dans l'humus et la couverture morte, éminemment hygroscopiques. De là, elles accèdent aux racines qui les absorbent partiellement, ou elles s'infiltrent dans les couches profondes. L'eau entraînée physiologiquement dans les tissus est presque totalement restituée à l'atmosphère par transpration foliacée. En Europe, on estime qu'une forêt adulte peut transpirer annuellement une tranche de $\mathrm{I} \mathrm{m}$. d'eau, et qu'elle restitue à l'atmosphère les $4 / 5$ de l'eau qu'elle reçoit.

La Forêt est donc un organe de perpétuelle "remise en travarl " des eaux continentales. Le sol boisé constitue ainsi de véritables " gisements de hourlle blanche " auxquels "l'Association forestière " assure au plus haut degré tous les éléments de pérennité.

Les autres armatures spontanées du sol jouissent, toutes proportions gardées, des mêmes propriétés hydrologiques; une grande partie de ces propriétés échappe à l'ensemble des cultures artificielles qui, abandonnées à elles-mêmes, évolueraient vers l'association spontanée locale.

Les observations tirées : $I^{\circ}$ de l'assèchement des régions marécageuses par la forêt (France, Italie, Algérie) : $2^{\circ}$ des constatations aéronautiques au-dessus des grands massifs forestiers (France); $3^{\circ}$ de la dépression des niveaux phréatıques dans les sols de forêts de plaine (Russie, France, Inde); $4^{\circ}$ du relèvement du niveau des nappes halophiles dans les régions déboisées (Algérie) confirment l'intensité de la "respiration" du sol par la Forêt.

Les déforestations pastorales, culturales et industrielles qui, au cours du $19^{\circ}$ siècle, et surtout en montagne, ont dénaturé l'armature végétale spontanée du sol, ont nécessairement eu leur répercussion météorologique sur la circulation aérienne des eaux, sur le régime des rivières, sur l'enneigement des écrans montagneux, sur l'ensemble des gisements de houille blanche. Le recul général de tous les glaciers européens est d'autant plus marqué qu'ils sont plus continentaux, plus soumis au fait humain de la dénudation.

L'insuffisance de la légıslation française en matière de protection de l'armature végétale spontanée du sol, son inaptitude actuelle au développement des gisements de houlle blanche, résultent des faits sommaires ci-après : la loi du 4 avril I882 sur la restauration des montagnes n'a aucun caractère préventif; $2^{\circ}$ le régime forestier doit abandonner aux déprédations de la "jouissance collective " (a) de grandes étendues forestières montagneuses qui, ailleurs qu'en France, seraient rigoureusement conservées comme forêts de protection (b) des millions d'hectares de landes banales, à ruissellement, dont un régime pastoral devrait réglementer strictement la jouissance; $3^{\circ}$ la loi du 18 juin J 859 n'entrave pas le défrichement des bois particuliers; $4^{\circ}$ la loi du 17 décembre 1902 est sans aucun effet sur les incendies des forêts, landes boisées ou nues.

Les réformes à opérer doivent s'inspirer des mesures prises par les nations voisines et surtout procéder de ces idées que, dans un grand Etat comme la France, les forêts ne sont point exclusivement matière fiscale, les montagnes ne constituent pas exclusivement des voisinages dangereux. Les unes et les autres sont, pour la masse croissante des clients des eaux et de la houille blanche, de puissants éléments de travail et de prospérité..... si l'on sait s'en servir.

\author{
I.-A. FABRE, \\ Inspecteur des Eaux et Forêts.
}

\section{NOTE AU SUJET DES COUPS DE BÉLIER}

\author{
Par M. le comte De Sparre
}

M. Alliévi a publié dans les numéros de janvier et mars de la Revue de Mécanique, une étude très remarquable à bien des points de vue, du phénomène des coups de bélier.

M. Alliévi part de l'étude du mouvement vibratoire de l'eau, et à première vue il ne semble pas faire d'hypothèses bien particulières, mais si les hypothèses introduites ne sont pas en évidence, elles n'en existent pas moins. Les principales de ces hypothèses sont les suivantes :

$1^{\circ} \mathrm{M}$. Alliévi identifie la vitesse de vibration de l'eau avec sa vitesse d'écoulement;

$2^{\circ}$ Il suppose que la seule vibration qui se produit est toujours la vibration fondamentale et il néglige les harmo. niques;

30 Il suppose que les réactions, soit de l'eau, soit du tuyau, se produisent comme à l'état statique.

Or, il est évident que, tout au moins au début du phéno. mène, les chocs des tranches liquides les unes contre les autres feront que la vitesse de vibration du liquide différera beaucoup de sa vitesse d'écoulement. De plus, ces chocs donneront naissance à des harmoniques qui pourront s'atté. nuer beaucoup par la suite, mais qui, pendant la période d'établissement du mouvement d'ensemble, celle que M. Alliévi appelle période du coup de bélier simple ou direct, pourront être absolument prédominantes sur la vibration fondamentale. Il faut remarquer enfin que pendant cette première période du mouvement, période pendant laquelle la pression varie très rapidement, les réactions, soit de l'eau, soit du tuyau, ne seront nullement celles qui se produiraient à l'état statique. Il y aura d'abord, à cause de l'inertie, un certain retard dans la compression du liquide et dans la dilatation du tuyau, retard qui produira une augmentation supplémentaire de pression, puis, tant par suite de cette augmentation de pression qu'en vertu de la vitesse acquise, la compression du liquide et la dilatation du tube dépasseront ce qu'elles auraient été à l'état statique. L'influence de ce phénomène s'atténuera presque complè. tement par la suite, une fois que, le mouvement s'étant propagé dans tout le tube, les variations de pressions seront beaucoup plus lentes. Toutefois, il semble que les hypothèses introduites par M. Alliévi ne sont guère admissibles pour la première période du mouvement.

Avant la théorie de M. Alliévi, une solution de cette 
question avait ćté donnée par M. Rateau. M. Rateau traite le mouvement du liquide dans son ensemble, en supposant que l'augmentation de pression, due au coup de bélier, croît uniformément depuis l'origine du tuyau jusqu'à son extrémité. Cette hypothèse est très acceptable une fois que le phénomène s'est propagé tout le long du tuyau, et si elle l'est moins pendant les premiers instants, celles qu'introduit M. Alllévi ne le sont, somme toute, guère plus.

Je remarquerai, d'ailleurs, que si on néglige la dilatation du tuyau et la compression du liquide, ce que l'on ne peut faire si on suppose une fermeture très brusque ou si on se propose d'étudier ce qui se passe après la fermeture de la vanne, mais ce que l'on peut admettre si la fermeture se fait dans un temps plus long que celui que M. Alliévi appelle la période du coup de bélier direct, la méthode de $M$. Rateau, en ne se bornant pas à l'intégrale approchée qu'il donne, mais en obtenant, ainsi que je l'indiquerai, l'intégrale exacte, fournit identiquement le même résultat que celle de M. Alliévi pour la valeur du coup de bélier. De plus, ainsi que je le ferai voir, elle fournit le résultat au moyen de formules très simples.

Je ne vois donc en réalité aucune raison, au point de vue de la rigueur, pour préférer une des méthodes à l'autre, mais en revanche celle de $M$. Rateau me parait plus propre à étudier certains côtés de la question, comme par exemple l'influence des tubes piézométriques que je me propose d'examiner.

Dans ce qui va suivre je me propose seulement de reprendre la méthode de M. Rateau pour le cas où l'on néglige la dilatation du tuyau et la compression du liquide, et de donner dans ce cas l'intégration complète des équations que M. Rateau n'avait donnée que d'une façon approchée. On obtient alors, ainsi que je l'ai dit plus haut pour le coup de bélier, un résultat qui coïncide avec celui obtenu par M. Alliévi, mais ce résultat se présente par la méthode que nous indiquons sous forme de formules très simples qui sont susceptibles de rendre, à ce que je crois, des services pour les applications. J'étudirai ensuite dans une seconde partie l'influence des tubes piézométriques en admettant encore que l'on néglige la dilatation du tuyau et la compression du liquide, ce qui ne peut être admis que si la fermeture n'est pas trop brusque. Je me propose de revenir plus tard sur cette question d'une façon plus complète, et en tenant compte de la compression du liquide et de la dilatation du tuyau.

\section{I}

\section{ETUDE DU COUP DE BÉLIER \\ LORSQU'ON NEGLIGE LA DILATATION DU TUYAU ET LA COMPRESSION DU LIQUIDE}

Dans ce cas, toutes les molécules du liquide sont supposées avoir la même vitesse à l'intérieur de la partie cylindrique de la conduite, de plus, puisque l'on néglige et la compression du liquide et la dilatation du tuyau on pourra appliquer le théorème des forces vives sans avoir à tenir compte du travail correspondant à ces deux phénomènes.

Appliquons donc le théorème des forces vives pendant le temps $d t$ à la portion du liquide comprise entre une section
$A_{0} B_{0}$ voisine du début de la conduite et une section $A_{1} B_{1}$ voisine du distributeur. Soit :

$L$ la longueur de la conduite entre $A_{0} B_{0}$ et $A_{1} B_{1}$.

$H$ la différence de charge nette entre $A_{0} B_{0}$ et $A_{1} B_{1}$.

$p_{0}$ la pression en $\mathrm{A}_{0} \mathrm{~B}_{0}$.

$p_{1}$ la pression en $\mathrm{A}_{1} \mathrm{~B}_{1}$.

(Nous supposerons que l'on puisse considérer $H$ comme constant) $\left(^{*}\right)$.

$V$ la vitesse de l'eau dans la conduite à un instant quelconque.

$c$ la vitesse de régime dans la conduite.

$S$ la section de la conduite,

$s$ celle du distributeur, lorsqu'il est entièrement ouvert, $s x$ cette ouverture a un instant quelconque,

$p_{0}+H(\mathrm{I}+\mathrm{Z})$ la pression en $\mathrm{A}_{1} \mathrm{~B}_{1}$ à un instant quelconque.

Le liquide compris à l'origine du temps entre $A_{0} B_{0}$ et $\mathrm{A}_{1} \mathrm{~B}_{1}$ est compris au bout du temps $d t$ entre $\mathrm{A}_{0}^{\prime} \mathrm{B}_{0}^{\prime}$ et $\mathrm{A}_{1}^{\prime} \mathrm{B}_{1}^{\prime}$ et la variation de sa force vive, puisque nous supposons toutes les molécules animées de la même vitesse $V$, sera :

$$
\frac{\pi L S}{g} V d V\left(^{* *}\right)
$$

$\pi$ étant le poids spécifique du liquide.

Pendant ce temps le travail des pressions sera :

$$
p_{0} S V d t-p_{1} S V d t=-\pi H(\mathrm{I}+Z) V d t
$$

puisque $\quad p_{1}=p_{0}+\pi H(\mathrm{I}+Z)$.

Le travail de la pesanteur et du frottement sera, puisque $H$ désigne la charge nette :

$$
\pi S V d t . H
$$

Puisque nous négligeons la compression du liquide et la dilatation du tube, le théorème des forces vives nous donne, en divisant l'équation obtenue par $\pi S V d t$ :

$$
\frac{L}{g} \frac{d V}{d t}=-Z H
$$

C'est la première équation de M. Rateau.

Pour la seconde équation dont il se sert, elle n'est qu'approchée et il est bon d'en donner une démonstration rigoureuse.

Appliquons pour cela le théorème des forces vives pendant le temps $d t$ à la partie du liquide comprise entre une section voisine et l'extrémité de la conduite cylindrique, du côté du distributeur et la section contractée du distributeur.

On aura, en désignant par $u$ la vitesse de l'eau à la section contractée du distributeur $\left(^{* * *}\right)$.

$$
\begin{gathered}
\frac{\pi S V d t}{2 g}\left(u^{2}-V^{2}\right)+\Sigma m v d v \\
=S V d t\left[p_{0}+\pi H(\mathrm{r}+Z)\right]-s x u d t \cdot p_{0}
\end{gathered}
$$

(*) La charge nette $H$ entre $A_{0} B_{0}$ et $A_{1} B_{1}$ est en réalité fonction de $V$, elle est en effet égale à la diftérence de niveau diminuée de la perte de charge, perte qui devient nulle pour $V=o$.

(**) En négligeant la différence entre la force vive de $A_{0} B_{0} A_{0}^{\prime} B_{0}^{\prime}$ et celle de $A_{1} B_{1} A_{1}^{\prime} B_{1}^{\prime}$, différence qui est infiniment petite du second ordre.

$(* * *)$ Nous supposons qu'il s'agisse d'une turbine sans réaction, de sorte que la pression dans la section contractée du distributeur est supposée la pression atmosphérique. 
$V$ désignant, comme plus haut, la vitesse dans la partie cylindrique, $m$ la masse d'une molécule comprise entre les deux sections considérées et $\nu$ sa vitesse. Mais, du moment que nous négligeons la compressibilité du liquide et la dilatation du tuyau, l'équation de continuité nous donne :

$$
S V=s u x
$$

et comme pour $x=\mathrm{r}$, on a, $c$ étant la vitesse de régime :

$$
V=c, \quad u=\sqrt{2 g H}
$$

on en déduit : $\quad S c=s \sqrt{2 g H}$

$$
\text { d'où : } \quad V=\frac{c u x}{\sqrt{2 g H}}
$$

de sorte que l'équation (2) donne :

$$
\frac{H V^{2}}{c^{2} x^{2}}\left(\mathrm{r}-\frac{c^{2} x^{2}}{2 g H}\right)=H(\mathrm{r}+Z)-\frac{\mathrm{r}}{\pi S V} \Sigma m \nu \frac{d \nu}{d t}
$$

Là, les diverses molécules qui figurent dans le dernier terme n'ont plus la même vitesse que celles qui remplissent la conduite cylindrique, mais, si on considère toutes les molécules d'une tranche comme ayant même vitesse et si $\sigma$ est la surface de cette tranche on aura :

$$
\sigma \nu=S V, \quad m=\frac{\pi \sigma d l}{g}
$$

et l'équation (3) devient :

$$
V^{2}\left(\mathrm{I}-\frac{c^{2} x^{2}}{2 g H}\right)=c^{2} x^{2}(\mathrm{I}+Z)-\frac{c^{2} x^{2}}{g H} \frac{d V}{d t} \Sigma \frac{S d l}{\sigma}
$$

Mais on a forcément :

$$
\frac{S}{\sigma}<\frac{S}{s}=\frac{\sqrt{2 g H}}{c}
$$

de sorte qu'en désignant par $\alpha$ un facteur plus petit que I et en remplaçant $d V$ par sa valeur (r) on aura :

$$
V^{\mathrm{q}}\left(\mathrm{I}-\frac{c^{2} x^{\mathrm{q}}}{2 g H}\right)=c^{2} x^{2}\left[\mathrm{I}+Z+\frac{a \alpha \sqrt{2 g H}}{c L} Z\right]
$$

a désignant la longueur de la conduite dans la partie considérée.

Mais $c x$ sera très petit par rapport à $2 g H$, la vitesse dans la conduite devant rester assez faible.

$$
\text { De même : } \quad \frac{\sqrt{2 g H}}{L}
$$

sera petit car $L$ sera presque toujours plus grand que $2 H$, de sorte que l'on a:

$$
\frac{\sqrt{2 g H}}{L}<\sqrt{\frac{g}{L}}
$$

Or, dans le cas qui nous occupe, $L$ sera toujours beaucoup plus grand que $g$, on pourra donc dans ces conditions prendre :

$$
V^{\mathrm{a}}=c^{2} x^{2}(\mathrm{I}+Z)\left(^{*}\right)
$$

C'est la seconde équation de M. Rateau, mais qui n'est applicable que si l'on suppose $g$ négligeable par rapport à $L\left({ }^{* *}\right)$.

(*) Donc $a=\Sigma d l$.

(*) Dans ces formules nous considérons $H$ comme constant, tandis qu'en réalité la perte de charge dépend de la vitesse, mais cette différence peut être négligée si la vitesse dans la conduite est faible.
Supposons maintenant que le mouvement de fermeture soit uniforme et posons, $T$ étant le temps de fermeture :

$$
x=\mathrm{I}-\frac{t}{T} \quad(5) \quad n=\frac{L c}{g H T}
$$

L'équation ( $\mathrm{I}$ ) deviendra :

$$
Z=\frac{n}{c} \frac{d V}{d x}
$$

et (4) donnera alors:

$$
V^{2}=c^{2} x^{2}\left(1+\frac{n}{c} \frac{d V}{d x}\right)
$$

Cette équation n'est pas linéaire, mais on peut l'intégrer de la façon suivante :

$\zeta$ étant une variable et $\alpha$ une constante arbitraire, posons: $\quad V=c x(\alpha+\zeta)$

L'équation (8) deviendra :

$$
\alpha^{2}+2 \alpha \zeta+\zeta^{2}=\mathrm{I}+n(\alpha+\zeta)+n x \frac{d \zeta}{d x}
$$

Posons de nouveau : $\quad \alpha^{2}-n \alpha-1=0$

cette équation déterminant $\alpha$. Nous prendrons, d'ailleurs, pour $\alpha$ la racine positive de cette équation en prenant par suite :

$$
\alpha=\frac{n+\sqrt{n^{2}+4}}{2}
$$

Mais $n$ est, en général, notablement plus petit que 2; on pourra par suite prendre le plus souvent :

$$
\alpha=\mathrm{I}+\frac{n}{2}+\frac{n^{2}}{8}
$$

$\alpha$ vérifiant la relation (I I), l'équation ( $\mathrm{o}$ ) devient :

d'où :

$$
(2 x-n) \zeta+\zeta^{2}=n x \frac{d \zeta}{d x}
$$

$$
\frac{\mathrm{I}}{n} \frac{d x}{x}=\frac{\mathrm{I}}{2 \alpha-n}\left[\frac{\mathrm{I}}{\zeta}-\frac{\mathrm{I}}{2 \alpha-n+\zeta}\right] d \zeta
$$

Intégrant et remarquant qu'en vertu de (9) pour $x=\mathrm{I}$ on a $\zeta=-(\alpha-1)\left(^{*}\right)$, on aura :

$$
x^{\frac{2 a-n}{n}}=-\frac{\alpha+\mathrm{I}-n}{\alpha-\mathrm{I}} \frac{\zeta}{2 \alpha-n+\zeta}
$$

d'où :

$$
\zeta=-\frac{(2 \alpha-n)(\alpha-1) x^{\frac{2 a-n}{n}}}{\alpha+\mathrm{I}-n+(\alpha-1) x^{\frac{2 a-n}{n}}}
$$

$\zeta$ étant connu, la formule (9) fera connaître $V$ et au moyen de la formule $(7)$ on aura ensuite :

$$
Z=n(\alpha+\zeta)+n x \frac{d \zeta}{d x}
$$

$\zeta$ étant négatif ainsi que $\frac{d \zeta}{d x}$, et $\zeta$ étant nul pour $x=0$, le maximum de $Z$ aura lieu pour $x=$ o et il sera égal à $n a$.

Dans le cas que nous considérons la valeur maximum du coup de bélier est donc :

$$
n H_{\alpha}=\frac{L c}{g T} \alpha
$$

(*) On $a$, en vertu de l'équation qui détermine $\alpha$ :

$$
\alpha>1+\frac{n}{2} \quad \alpha>n \quad \alpha<1+n
$$


où $\alpha$ est donné par la formule (I 1$)\left(^{*}\right)$. Si $n$ est inférieur à $\mathrm{I}$, ce qui aura lieu très généralement, on pourra prendre pour $\alpha$ la valeur (I I)' et on aura alors, pour le maximum du coup de bélier :

$$
n H_{\alpha}=\frac{L c}{g T}\left(\mathrm{I}+\frac{n}{2}+\frac{n^{2}}{8}\right)
$$

On aura, d'ailleurs, avec la même approximation, en prenant pour $\alpha$ la valeur (I I)' :

$$
\zeta=-\frac{n}{2} \frac{\left(\mathrm{I}+\frac{n^{2}}{8}\right)\left(\mathrm{I}+\frac{n}{4}\right) x^{\mathrm{h}}}{\mathrm{I}-\frac{n}{4}+\frac{n^{2}}{\mathrm{I} 6}+\frac{n}{4}\left(\mathrm{I}+\frac{n}{4}\right) x^{\mathrm{h}}}
$$

où l'on a posé, pour simplifier l'écriture,

$$
h=\frac{2}{n}+\frac{n}{4}=\frac{2}{n}\left(\mathrm{r}+\frac{n^{2}}{8}\right)
$$

Le plus souvent $n$ sera assez petit pour que l'on puisse négliger $n^{2}$, d'autant plus qu'il s'agit d'un résultat qu'il n'est pas nécessaire de connaître avec une grande approximation.

D’ailleurs la valeur relative du coup de bélier étant $n \alpha$, comme $\alpha>\mathrm{I}+\frac{n}{2}$, pour $n=\frac{\mathrm{I}}{2}$, cette valeur relative du coup de bélier sera supérieure à $1 / 2\left(1+\frac{1}{4}\right)$ ou à 62,5 p. I00; or, on aura, en général, intérêt à ce que cette valeur ne soit pas atteinte et, par suite, à faire en sorte que $n$ soit inférieur à $\frac{\mathrm{r}}{2}$, donc $\frac{n^{2}}{8}$ inférieur à $\frac{\mathrm{r}}{32}$.

On aura alors, en négligeant $\frac{n^{2}}{8}$ :

$$
\begin{gathered}
V=c x\left[\mathrm{I}+\frac{n}{2}\left(\mathrm{I}-x^{\frac{2}{n}}\right)\right] \\
Z=n\left(\mathrm{I}+\frac{n}{2}\right)\left(\mathrm{I}-x^{\frac{2}{n}}\right) \\
u=\sqrt{2 g H}\left[\mathrm{I}+\frac{n}{2}\left(\mathrm{I}-x^{\frac{2}{n}}\right)\right]
\end{gathered}
$$

On arrive ainsi à des formules très simples qui seront très suffisamment approchées, lorsque la rapidité de la fermeture ne sera pas trop grande.

Il est facile d'obtenir de plus, dans ce cas, la force vive maximum du jet.

La force vive du jet est proportionnelle à $x u^{3}$, et comme pour $x=\mathrm{I}, u=\sqrt{2 g H}$, la valeur relative $y$ de la force vive du jet est donnée par la formule :

$$
y=\frac{u^{3} x}{(2 g H)^{\frac{3}{2}}}=x\left[\mathrm{I}+\frac{n}{2}\left(\mathrm{I}-x^{\frac{2}{n}}\right)\right]^{3}
$$

Cette expression sera maximum pour :

d'où :

$$
1+\frac{n}{2}\left(1-x^{\frac{2}{n}}\right)-3 x^{\frac{2}{n}}=0
$$

$$
x=\left[\frac{1+\frac{n}{2}}{3+\frac{n}{2}}\right]^{\frac{n}{2}}
$$

(") Cette valeur est exactement celle donnée par M. Alliévi pour le cas où la fermeture a lieu dans un temps supérieur à ce qu'il appelle temps du coup de bélier simple. ou, avec la même approximation que plus haut :

$$
\begin{gathered}
x=\left[\frac{\mathrm{I}}{3}\left(\mathrm{I}+\frac{n}{3}\right)\right]^{\frac{n}{2}} \\
x_{\mathrm{m}}=\left[\frac{\mathrm{I}}{3}\left(\mathrm{r}+\frac{n}{3}\right)\right]^{\frac{n}{2}}
\end{gathered}
$$

On aura en portant cette valeur de $x$ dans l'expression de $y$ pour la valeur relative de la force vive maximum comparée à la force vive de régime :

$$
y_{\mathrm{m}}=\left[\mathrm{I}+\frac{n}{3}\left(\mathrm{I}-\frac{n}{6}\right)\right]^{3}\left[\frac{\mathrm{I}}{3}\left(\mathrm{x}+\frac{n}{3}\right)\right]^{\frac{n}{2}}
$$

ou toujours avec la même approximation :

$$
y_{\mathrm{m}}=\left(\mathrm{I}+\frac{n}{3}\right)^{3}+\frac{n}{2}\left(\frac{\mathrm{I}}{3}\right)^{\frac{n}{2}}\left(^{*}\right)
$$

\section{II}

\section{INFLUENCE DES TUBES PIÉZOMÉTRIQUES}

\section{LORSQU'ON NÉGLIGE LA DILATATION DE LA CONDUITE} ET LA COMPRESSIBILITÉ DU LIQUIDE

Cette question des tubes piézométriques ou des cheminées d'aération semble préoccuper assez les constructeurs à l'heure présente; nous allons voir que si les cheminées de grand diamètre, telles que celles installées par la Société de Fure-et-Morge à son usine de Champ, peuvent donner de très bons résultats et supprimer presque complètement le coup de bélier; au lieu de cela les tubes piézométriques de petit diamètre ne les atténuent presque pas. Nous supposerons que l'on se trouve dans le cas de la fermeture uniforme et que l'on a comme plus haut :

$$
x=1-\frac{t}{T}
$$

Nous conserverons, d'ailleurs, les mêmes notations que plus haut, mais nous désignerons, de plus, par $\sigma$ la section du tube piézométrique ou de la cheminée d'aération, dont nous supposerons la section uniforme, par $w$ la vitesse dans ce tube, par $L_{1}$ sa longueur totale et par $H_{1}$ la charge nette à la partie inférieure du tube piézométrique.

Cette charge $H_{1}$ est égale à $H$ lorsque la surpression $Z$ est nulle; d'une façon générale nous poserons :

$$
\left.H_{1}=H(\mathrm{I}+\mu . Z){ }^{* *}\right)
$$

$\mu$ étant un facteur constant que nous déterminerons après coup.

Considérons d'abord le tuyau depuis son origine jusqu'au début du tube piézométrique, nous aurons :

$$
\frac{d V}{d t}=-\frac{Z g H}{L}
$$

ou en posant toujours : $\quad n=\frac{c L}{g T H}$

et tenant compte de (I): $\frac{d V}{d x}=\frac{c Z}{n}$

(*) Pour $n=1 / 2$ on trouverait : $x_{\mathrm{m}}=0,79 \quad y_{\mathrm{m}}=\mathrm{r}, 25$.

Pour $n=1 / 4$ on trouverait : $x_{\mathrm{m}}=0,88 \quad y_{\mathrm{m}}=1,12$.

$\left(^{* *}\right)$ Ce n'est là qu'une approximation, somme toute, assez grossière, mais elle sera suffisante pour l'objet que nous avons en vue. 
L'équation de continuité nous donnera d'ailleurs, "étant toujours la vitesse au distributeur :

$$
S V=s x u+\sigma w
$$

Mais nous avons encore, comme dans le cas précédent:

$$
\text { et } \quad u=\sqrt{2 g H(\mathrm{I}+Z)}
$$

on en conclut : $V=c x \sqrt{1+Z}+\frac{\sigma}{S} \mathfrak{w}$

Appliquons maintenant le théorème des forces vives au mouvement de l'eau dans le tube piézométrique pendant le temps $d t$.

La pression à la partie inférieure de ce tube est $p_{0}+H$ $(\mathrm{I}+Z)$, le travail des pressions sera done:

$$
\pi \sigma H(\mathrm{r}+Z) w d t
$$

Le travail de la pesanteur et du frottement $\left(^{*}\right)$ sera :

$$
-\pi \sigma H_{1} v d t=-\pi \sigma H(\mathrm{r}+\mu Z) z d t
$$

puisque nous avons désigné par $H_{1}$ la charge augmentée de la perte de charge.

Le théorème des forces vives nous donnera donc après suppression du facteur commun :

$$
\frac{L_{1}}{g} \frac{d w}{d t}=H Z(\mathrm{r}-\mu)
$$

ou en tenant compte de (1):

$$
\frac{d w}{d x}=-\frac{g H T}{L_{1}} Z(\mathrm{I}-u)
$$

ce qui peut s'écrire :

$$
\frac{d w}{d x}=-\frac{c}{n} \frac{L}{L_{1}} Z(\mathrm{1}-\mathrm{w})
$$

Si nous éliminons $Z$ entre (2) et (4) nous aurons:

$$
\frac{d w}{d x}+\frac{L}{L_{1}}(\mathrm{i}-\text { p. }) \frac{d V}{d x}=0
$$

ou en intégrant et remarquant que pour $x=\mathrm{I}$ on a : $v=0$ et $V=c$ :

$$
w+\frac{L}{L_{1}}(\mathrm{r}-\mu)(V-c)=\mathrm{o}
$$

d'où : $\quad w=\frac{L}{L_{1}}(1-\mu)(c-V)$

$\mathrm{Si}$-on porte cette valeur de $w$ dans (3) et si on pose de plus : $\lambda=\frac{\sigma L}{S L_{1}}$, nous aurons :

$$
V[\mathrm{I}+\lambda(\mathrm{I}-\mu)]=\lambda(\mathrm{I}-\mu) c+c x \sqrt{\mathrm{I}+Z}
$$

$\mathrm{Si}$ alors nous posons $\alpha$ désignant une constante et $\zeta$ une nouvelle variable.

$$
(\alpha+\zeta) c x=V[\mathrm{I}+\lambda(\mathrm{I}-\mu \mathrm{u})]-\lambda c\left(\mathrm{I}-\mathrm{p}_{\mathrm{j}}\right)
$$

d'où l'on déduit :

$$
\frac{d V}{d x}=\frac{c}{1+\lambda(1-\mu)}\left[\alpha+\zeta+x \frac{d \zeta}{d x}\right]
$$

(*) Il y a lieu ici de tenir compte du travail du frottement, parce que le mouvement de l'eau dans le tube piézométrique peut être rapide.
L'équation (6) élevée au carré, s'écrira :

$$
(x+\zeta)^{2}=\mathrm{I}+Z=\frac{n}{c} \frac{d V}{d x}+\mathrm{I}
$$

Posons de nouveau :

$$
m=\frac{n}{1+\lambda(\mathrm{I}-\mu)} \quad \text { (8) } \quad \alpha^{2}-m \alpha-\mathrm{I}=0
$$

En prenant pour $\alpha$ la racine positive, donc :

$$
\alpha=\frac{m+\sqrt{m^{8}+4}}{2}
$$

Ce qui, si on suppose $m<2$ et si on développe, pourra s'écrire, en se bornant aux premiers termes :

$$
\alpha=\mathrm{I}+\frac{m}{2}+\frac{m^{2}}{8}
$$

Nous aurons alors :

$$
\zeta(\zeta+2 \alpha-m)=m x \frac{d \zeta}{d x}
$$

C'est l'équation de la première partie où $n$ est seulement remplacé par $m$, comme d'ailleurs on a en vertu de (7) $\zeta=-(\alpha-\mathrm{I})$ pour $x=\mathrm{I}$ la valeur de $\zeta$ sera donc la même que lorsqu'il n'y a pas de tube piézométrique, sauf le remplacement de $n$ par $m$, il viendra donc:

$$
\zeta=-\frac{(2 \alpha-m)(\alpha-1) x^{\frac{2 a-m}{m}}}{\mathrm{I}+\alpha-m+(\alpha-1) x^{\frac{2 a-m}{m}}}
$$

et si nous négligeons les termes en $m^{2}$ ce qui conduit à prendre :

$$
\zeta=-\frac{m}{2} x^{\frac{2}{m}} \quad \alpha=\mathrm{I}+\frac{m}{2}
$$

nous aurons :

$V=\frac{c}{\mathrm{I}+\lambda(\mathrm{I}-\mu)}\left[\lambda(\mathrm{I}-\mu)+x\left[\mathrm{I}+\frac{m}{2}\left(\mathrm{I}-x^{\frac{3}{m}}\right)\right]\right]$

Puis les relations :

$$
Z=\frac{n}{c} \frac{d V}{d x} \quad m=\frac{n}{\mathrm{I}+\lambda(\mathrm{s}-\mu)}
$$

donneront : $\quad Z=m\left(\mathrm{x}+\frac{m}{2}\right)\left(\mathrm{I}-x^{\frac{2}{m}}\right)$

On déduira de même de la relation (5):

$w=\frac{L}{L_{1}} \frac{c(\mathrm{I}-\mu)}{\mathrm{I}+\lambda(1-\mu)}\left[1-x\left[\mathrm{I}+\frac{m}{2}\left(\mathrm{I}-x^{\frac{2}{m}}\right)\right]\right]$

On aura enfin, en vertu de (3) :

$$
u=\sqrt{2 g H}\left[\mathrm{I}+\frac{m}{2}\left(\mathrm{I}-x^{\frac{2}{m}}\right)\right] c x
$$

Il nous reste à déterminer une valeur convenable pour w... Pour cela nous la choisirons de façon que pour $x=0$ l'on ait $H(1+\mu . Z)=H_{1}$.

Mais $H_{1}$ est égal à $H$ augmenté de la différence de niveau $a$ entre la charge nette $H$ et le niveau de déversement, plus la perte de charge qui est égale à $\frac{4}{L} L_{1} b w_{1}^{0}\left(^{*}\right)$ en adoptant les coefficients de Darcy :

(*) Avec $b=0,000507+\frac{0,00001294}{D}$ 
$w_{1}$ étant la valeur de $w$ pour $x=0$; on aura done, puisque pour $x=0$ :

$$
Z=Z_{1}=m\left(1+\frac{m}{2}\right)
$$

$$
H\left[\mathrm{I}+\mu \cdot m\left(\mathrm{I}+\frac{m}{2}\right)\right]=H+a+\frac{4}{D} L_{1} b w_{1}{ }^{2}
$$

mais : $\quad w_{1}=\frac{L}{L_{1}} \frac{c(\mathrm{I}-\mu)}{\mathrm{I}+\lambda(1-\mu)}$

De sorte qu'en remarquant que :

$$
m=\frac{n}{\mathrm{I}+\lambda(\mathrm{I}-\mu)}
$$

notre équation, si on chasse les dénominateurs, pourra s'écrire :

$$
\left.\begin{array}{c}
n \mu\left[\mathrm{I}+\lambda(\mathrm{I}-\mu)+\frac{n}{2}\right] \\
=\frac{a}{H}[\mathrm{I}+\lambda(\mathrm{I}-\mu)]^{2}+\frac{4 L^{2} b}{H D L_{1}} c^{2}(\mathrm{I}-\mu)^{2}
\end{array}\right\}
$$

Nous nous bornerons à examiner deux cas :

$I^{n}$ La cheminée d'aération a un diamètre sensiblement égal à celui de la conduite, dans ce cas :

$$
\lambda=\frac{\sigma L}{S L_{1}}
$$

est un très grand nombre puisque nous supposons qu'il s'agisse d'une conduite de grande longueur et que $L_{1}$ est au lieu de cela sensiblement égal à $H+a$.

Dans ce cas nous écrirons l'équation (I6) :

$$
\begin{gathered}
\frac{n \mu}{1+\lambda(1-\mu)}\left[\mathrm{I}+\frac{\mathrm{I}}{2} \frac{n}{\mathrm{I}+\lambda(\mathrm{I}-\mu)}\right] \\
=\frac{a}{H}+\frac{4 L^{2} b}{D H L_{1}} \frac{c^{2}(\mathrm{I}-\mu)^{2}}{[\mathrm{I}+\lambda(1-\mu)]^{2}}
\end{gathered}
$$

Si alors $\mu$ n'est pas lui-même très voisin de $\mathrm{I}$, on pourra négliger $\mathrm{I}$ devant $\lambda(\mathrm{I}-\mu)$ et notre équation deviendra :

ou comme :

$$
\frac{n \mu}{\lambda(\mathrm{I}-\mu)}=\frac{a}{H}+\frac{4 b L^{2}}{D H L_{1}} \frac{c^{2}}{\lambda^{2}}
$$

$$
\begin{gathered}
\lambda=\frac{\sigma L}{S L_{1}} \\
\frac{n \mu}{\lambda(\mathrm{I}-\mu)}=\frac{a}{H}+\frac{4 b S^{2}}{D \sigma^{2}} \frac{L_{1}}{H}
\end{gathered}
$$

Mais comme par hypothèse $S$ diffère peu de $\sigma$ et $L_{1}$ de $H$ on pourra, vu la petitesse de $b$, négliger le dernier terme et on aura alors :

$$
\text { d'où : }
$$$$
\text { w. }\left(n+\frac{\lambda a}{H}\right)=\frac{\lambda a}{H}
$$

$$
\mu=\frac{\mathrm{r}}{\mathrm{r}+\frac{n H}{\lambda a}}
$$

ou, si on suppose que $H$ n'est pas assez grand pour que $\frac{n H}{\lambda a}$ ne soit pas petit par rapport à l'unité, nous pourrons prendre :

$$
\mu=\mathrm{r}-\frac{n H}{\lambda a}
$$

d'où :

$$
\lambda(\mathrm{I}-\mathrm{p})=\frac{n H}{a}
$$

et l'on aura, en négligeant $\frac{m^{2}}{2}$ qui est ici très petit :

$$
Z_{1}=m=\frac{n}{\mathrm{I}+\frac{n H}{a}}
$$

ou, a étant petit par rapport à $H: Z_{1}=\frac{a}{H}$

On voit que, dans ce cas, le coup de bélier $H Z_{1}=a$ se réduit à la différence entre la charge nette et la hauteur de déversement.

Donc une cheminée d'aération de diamètre à peu près égal à celui de la conduite supprime presque complètement le coup de bélier, et le réduit à la différence entre le niveau de déversement et à la charge.

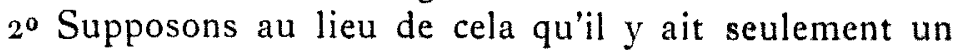
tube piézométrique de faible diamètre et admettons, pour fixer les idées, que le diamètre de ce tube piézométrique soit au plus égal au dixième de celui de la conduite.

On aura alors : $\quad \frac{\sigma}{S} \leqslant \frac{1}{100}$

et si nous supposons que $L$ ne dépasse pas too $L_{1}, \lambda$ sera au plus égal à $\mathbf{r}$.
Alors à cause du facteur :
$\frac{4 L^{2}}{H L_{1}}$

qui diffère peu de $\frac{4 L^{2}}{L^{2}}$, et qui est par suite très grand, puisque nous supposons qu'il s'agit d'une conduite de grande longueur, le dernier terme dans le second membre de (I6) sera prédominant. $\mathrm{Si}$ nous supposons par exemple $L>50 L_{1}$, ce facteur dépassera io ooo, de sorte que l'on aura : $\quad \frac{4 b L^{2}}{D H L_{1}}>\frac{5}{D}$

Comme d'ailleurs nous supposons le diamètre du tube piézométrique inférieur au dixième de celui de la conduite, $D$ ne pourra dépasser notablement om 25 et nous aurons :

$$
\frac{4 B L^{2}}{D H L_{1}}>20
$$

Nous pourrons donc dans le second membre de (16) négliger le premier terme devant le dernier et écrire cette équation :

$\left[\frac{4 L^{2} b}{D H L_{1}} c^{2}+n \lambda\right](1-\mu)^{2}+n\left(1+\frac{n}{2}-\lambda\right)(1-\mu)-n\left(1+\frac{n}{2}\right)=0$

D'après ce que nous venons de dire dans le cas actuel on a : $\quad \lambda<1 \quad \frac{4 b L^{2}}{D H L_{1}}>20$

Comme d'ailleurs $c$ sera, en général, supérieur à $\mathrm{I}$ et que l'on devra faire en sorte que $n$ ne dépasse pas $I$, on pourra prendre, avec une approximation suffisante :

$$
\mathrm{r}-\mathrm{u}=\sqrt{\frac{n+\frac{n}{2}}{\frac{4 L^{2} b c^{4}}{D H L_{1}}}}
$$

et on en conclut : $\quad 1-\mu<\sqrt{\frac{0,625}{20}}<0,177$

et par suite aussi : $\quad \lambda(1-\mu)<0,177$

d'où : $\quad m=\frac{n}{1+\lambda(1-\mu)}>\frac{n}{1,177}$ 


\section{donc sensiblement : $\quad m>0,85 n$}

On voit donc que, dans ce cas, l'existence du tube piézométrique ne réduirait le coup de bélier que de moins de $15 \%$ o.

On peut donc en conclure, ainsi que nous l'avons annoncé, que si une cheminée d'aération de grande section supprime presque entièrement le coup de bélier $\left(^{\star}\right)$, un tube piézométrique de faible dimension produit au lieu de cela un effet presque nul.

Dans le cas général, il faudrait calculer la valeur de $\mu_{\mathrm{au}}$ moyen de l'équation ( 16 ).

En résumé, dans le cas où il y a un tube piézométrique, le coup de bélier est fourni par la formule :

où :

$$
H Z_{1}=H m\left(\mathrm{r}+\frac{m}{2}\right)
$$

$m=\overline{\mathrm{i}+\lambda(\mathrm{I}-\mu)}$

$n$ ayant la même valeur que lorsqu'il n'y a pas de tube piézométrique : $\quad n=\frac{L c}{g T H}$

$$
\text { et où de plus : } \quad \lambda=\frac{\sigma L}{S L_{1}}
$$

- étant la section du tube piézométrique supposée constante, $L_{1}$ la longueur de ce tube et $\mu$ étant d'ailleurs la racine comprise entre o et $\mathrm{I}$ de l'équation ( 16 ) que l'on peut écrire $\left(^{* *}\right)$ :

$$
\begin{gathered}
{\left[\frac{4 L^{2} b}{H D L_{1}} c^{2}+n \lambda+\frac{a}{H} \lambda^{2}\right](\mathrm{I}-\mu)^{2}} \\
+\left[n\left(1+\frac{n}{2}\right)-n \lambda+\frac{2 a}{H} \lambda\right](\mathrm{I}-\mu)-n\left(\mathrm{I}+\frac{n}{2}\right)+\frac{a}{H}=o
\end{gathered}
$$

Dans cette formule :

a désigne la différence entre la charge et le niveau de déversement et $D$ le diamètre du tube piézométrique, de plus :

$$
b=0,000507+\frac{0,00001294}{D}
$$

Si le tube piézométrique ou la cheminée n'était pas de section constante, il serait facile, en suivant la même méthode, d'étendre les formules à ce cas, mais je ne crois pas devoir insister plus longuement pour ne pas augmenter outre mesure les dimensions de cette note, me réservant, d'ailleurs, de revenir sur cette question.

$N . B$. - Nous avons supposé que la perte de charge dans le tube piézométrique était uniquement due au frottement de l'eau contre les parois du tube, si ce tube n'était pas raccordé convenablement à la conduite, il y aurait un terme représentant la perte de charge par suite du changement brusque de section et du changement de direction dans le mouvement de l'eau à son entrée qu'il faudrait ajouter, et cela diminuerait encore d'autant l'effet du tube piézométrique.

\section{$\mathrm{C}^{\text {te }}$ De Sparre.}

(*) Dans le cas oú les hypothèses que nous avons faites sont admis sibles, c'est-à-dire, si la vitesse de fermeture n'est pas trop brusque.

$\left.{ }^{* *}\right)$ Cette équation contient toujours une $\mathrm{tacine}$ entre o et 1 parce que pratiquement $a<n$.

\section{LES CHEMINS DE FER TRAINSPYRÉNÉENS}

La commission internationale chargée d'étudier la question a terminé ses travaux et les deux gouvernements ont signé la convention qui marque le premier pas dans la réalisation de ces voies ferrées si longtemps attendues, si ardemment désirées. Il ne manque plus que les sanctions parlementarres et... les capitaux nécessarres, mais il y a lieu de croire qu'on obtiendra les uns et les autres.

Quoiqu'il en soit, en France comme en Espagne, peut-être plus en Espagne qu'en France, les esprits sont surchauffés L'opinion publique s'agite et tandis que les Aragonais craignent que le projet de Canfranc soit sacrifié aux deux autres, multiplient les démarches, rédigent des pétitions, les Catalans s'organisent pour exercer une action méthodique qui assure la réalisation de leurs vœux.

C'est ainsi qu'une importante réunion vient d'avoir lieu, le I4 de ce mois, à l'Hôtel de Ville de Puigcerdà, sous la présidence de M. le Maire de cette localité. Parmi les assistants, figuraient MM. Barrère et Aris, conseillers du canton de Saillagouse, ainsi que MM. les Maires des communes enclavées dans les Cerdagnes française et espagnole.

Trois commissions d'études ont été nommées :

La Commission de la Cerdagne Française, dont font partie MM. Emmanuel Brousse, conseiller général de Perpignan Jean Barrère-Balle et Michel Aris, conseillers d'arrondissement ;

La Commission de Puigcerdà, présidée par M. le maire Pedro Puig, assisté de MM. José M. Marti et Armengol Ferrer y Salo et de M. Eugenio Esteve y Deulofeu, secrétaire ;

La Commission de Barcelone, composée de MM. Salvador Andreu, Agustin Manaut, Francisco Simon et Rafael Gay de Montella, secrétaire.

D'autre part, tous les maires de Catalogne, invités par le maire de Barcelone, se réunissaient, le $\mathrm{I} 7$, à la mairie de cette ville et y prenaient d'importantes décisions, qu'on lira plus loin dans le compte rendu que nous donnons de cette réunion.

En résumé, tout le monde paraît animé de la meilleure volonté. Il n'y a plus qu'à attendre les événements et à faire des vœux pour que ce mouvement si bien commencé solt autre chose qu'un feu de paille.

Le moment nous semble bien choisi pour jeter un coup d'œil en arrière et aussi dire quelques mots des trois lignes en projet.

Quand on étudie la carte des chemins de fer espagnols on ne peut manquer d'être frappé du fait que, sur une frontière de 450 kilomètres, deux points seulement servent à assurer la communication des voies ferrées de l'Espagne avec celles de la France et, par elle, de toute l'Europe. Irun et Port-Bou, situés à chacune des extrémités de la chaîne des Pyrénées, sont les uniques stations-frontières des chemins de fer francoespagnols. La cordillère qui s'élève entre ces deux points forme une barrière sinon infranchissable, du moins infranchie, qui isole tout une partie de la Péninsule du reste de l'ancien continent.

On ne peut alléguer qu'il y a des moyens de communication par les routes de la montagne; car celles qui existent, peu nombreuses et en mauvais état, sont interceptées en hiver par les neiges et l'on doit reconnaître que le trafic international moderne s'accommode mal de la monture du muletier ou de la carriole du messager.

Diverses difficultés se sont opposées jusqu'à présent à la réalisation de quelques-uns - ou même d'un seul - des multiples projets de chemins de fer transpyrénéens qui ont vu le jour. Parfois on a invoqué le souci de la défense nationale; le plus souvent on s'est effrayé de la dépense et des difficultés du percement des Pyrénées; enfin, on a mis en a vant la nécessité d'harmoniser les convenances et les intérêts réciproques de la France et de l'Espagne. 